\title{
Comparison of perforator location in dynamic and static thermographic imaging with Doppler ultrasound in breast reconstruction surgery
}

\author{
by Sz. Kołacz*, M. Moderhak**, J. Jankau* \\ * Department of Plastic Surgery, Medical University of Gdańsk, ul. Debinki 7, 80-211, Gdańsk, \\ skolacz@gmail.com \\ ** Department of Biomedical Engineering, Gdańsk University. of Technology, 80-233, \\ Narutowicza Str., Gdańsk, Poland, matmod@biomed.eti.pg.gda.pl
}

Acknowledgement: This work has been partially supported by Statutory Funds of Electronics, Telecommunications and Informatics Faculty, Gdansk University of Technology

\begin{abstract}
This paper compares the effectiveness of the dTnorm and t90_10 parametrizations in dynamic thermography for imaging location of perforators in TRAM flaps in the intraoperative period. The results were compared with the location detected in a Doppler ultrasound examination. Cold and heat stimulation was used in dynamic thermography. Additionally, these results were compared with static thermography. The study was conducted on a group of 10 female patients after breast reconstruction.
\end{abstract}

\section{Introduction}

One of the most common plastic surgery methods used in managing integumental deficits is reconstruction with free flaps or pedicled flaps, for instance a musculocutaneous TRAM flap. Despite the fact that its anatomy is well-explored [1] its partial necrosis may develop due to blood circulation disorders. In the case of free flaps, preoperative planning is crucial, when vascular perforators are marked within the projection of the planned flap. The perforator which has the largest perfusion or the most convenient spatial location will be used for microsurgical anastomosis, and consequently for nourishing the whole flap. The final choice is made intraoperatively or after dissection. The redundant perforators are ligated and excised, and the main vessel is microsurgically anastomosed to recipient vessels. Pedicled TRAM flaps, in turn, seem to be a perfect model for investigating various kinds of imaging tests due to the flap anatomy. In contrast to DIEP (deep inferior epigastric perforator) flaps, they may include a number of perforators, which offers an opportunity for comparison of their images on subsequent days. It has to be noted here that because of the vascular pedicle blood supply to perforators in a pedicled flap is constant and the lack of microsurgical anastomosis prevents coagulation on the suturing material.

There are a number of methods of locating perforating vessels in the flap, such as CT angiography, laser Doppler, angiography or thermography. In order to be considered ideal in clinical conditions, a method should meet the following conditions of: non-invasiveness, simplicity, repeatability, ability of intraoperative assessment, and low cost [2].

\section{Problems}

Adequate preoperative planning is an essential part of reconstructive surgery. Considering its anatomy, a TRAM flap is divided into four parts (zones). Zone I and II zones are well vascularized and thus used in breast reconstruction. Zones III and IV are resected because of the poor intraoperative vascularization [3].

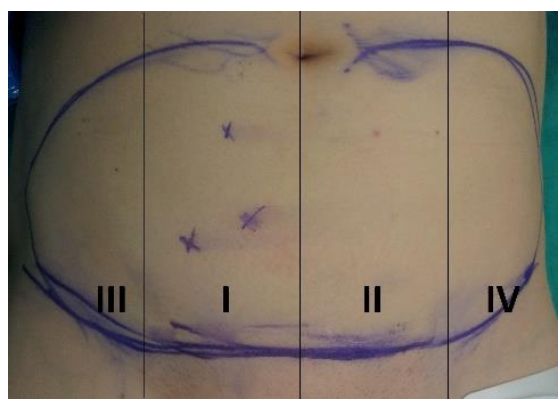

Fig. 1. TRAM flap zones

The division into zones is not specifically determined and its proper identification depends on the experience of the surgeon. The aim of the study was to compare perforator location as determined in dTnorm and t90_10 images in cold and heat stress dynamic thermography and in static thermography with Doppler ultrasound in the preoperative 


\subsection{1/qirt.2016.059}

planning period, and intra- and postoperatively on subsequent days, considering the fact that the relationship between the temperature of the skin and its blood supply is documented in literature $[4,5,6,7]$.

\section{Methods and results}

The study involved a group of 10 patients undergoing TRAM flap breast reconstruction. All treatments were provided by the same operating team. Each patient was examined preoperatively, intraoperatively and directly postoperatively, as well as at 1 and 7 days after the surgery. Each examination followed the protocol requiring static and dynamic thermography dTnorm and t90_10 with the stimulation with hot and cold air stream [8].

Each operated patient underwent a thermographic examination performed in compliance with the same standards with FLIR A320G camera with spatial resolution of $320 \times 240$ pixels and thermal resolution of $0.08 \mathrm{~K}$. The camera was positioned at a distance of $70 \mathrm{~cm}$ from the studied flap and perpendicularly to its maximum mound. The first imaging examination was performed before the surgery, the second one - after the dissection of the musculocutaneous flap and after excision of its excesses, the third one - immediately after suturing flap in the recipient region, and the follow-up ones - at 1 and 7 days after the surgery. Each examination consisted of the following steps:

- $\quad$ preoperative localization of perforators with a handheld Doppler ultrasound probe and marking with permanent marker

static thermal imaging

- $\quad$ dynamic thermal imaging with high energy stimulation of the flap for 60 seconds and monitoring changes in temperature over the next 180 seconds. A set of 20 halogen lamps (1000W) provided stimulation.

dynamic thermal imaging with low energy stimulation of the flap for 60 seconds and monitoring changes in temperature over the next 180 seconds. The lobe was stimulated with a stream of cooled air from a TC25 Titan-Cool air conditioner of 2,6kW cooling capacity; manufacturer: Premiair Appliances Ltd, United Kingdom..

The locations of preoperatively marked hot-spots were compared with pre, intra and postoperative thermographic examinations. We are aware that a perforator could be accidentally overlooked in a flap in a Doppler ultrasound test that is in this study we marked in zone I three places whose acoustic signal could indicate a perforator. Then, after collecting photographic documentation, we performed a static and dynamic thermographic test, and the images were mapped onto one another and compared.
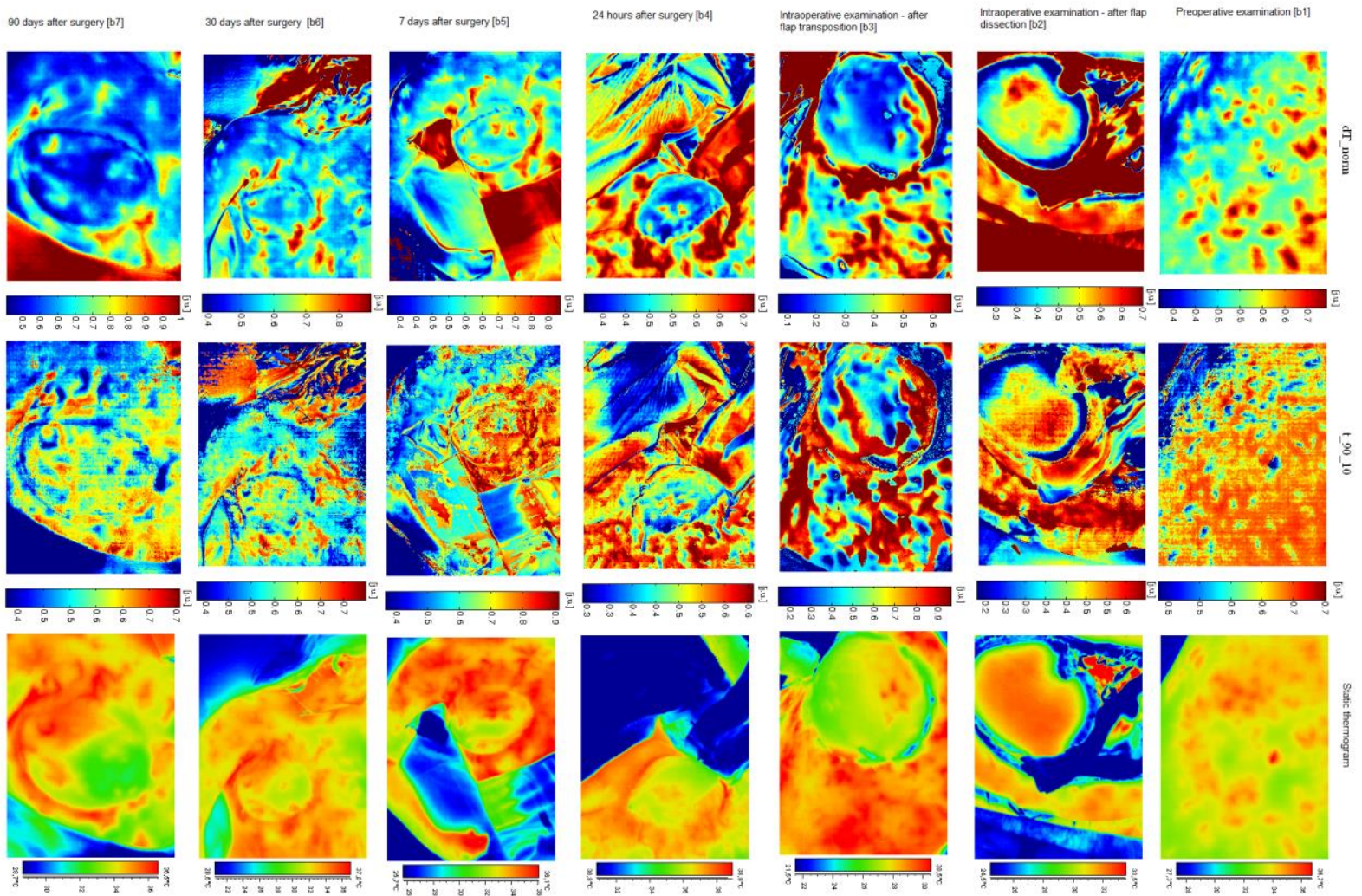

Fig. 2. Patient number 5 IPSI. Static thermography and ADT after cooling 

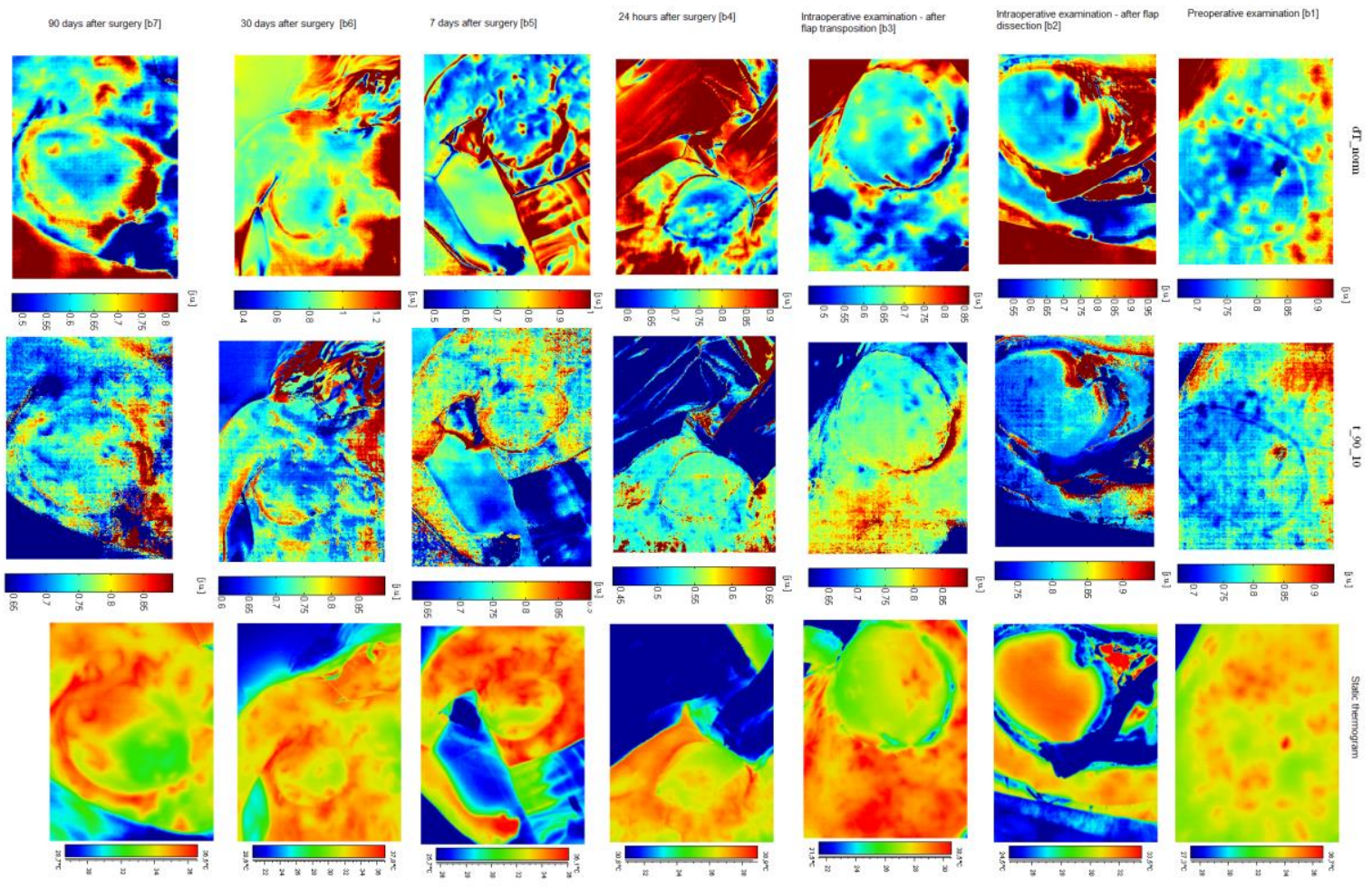

Fig. 3. Patient number 5 IPSI. Static thermography and ADT after heating

There are available descriptions of perforator mapping for reconstructive procedures with DIEP flaps. They are used for selecting and utilizing a perforator for microsurgical anastomosis with subsequent record of temperature change in time to evaluate the patency of the microsurgical anastomosis. Because there is no available literature reporting a description of static and heat or cold stress dynamic thermography with Doppler ultrasound in perforator localization in various perioperative periods in pedicled flaps in people, we cannot refer to them.

None of the patients developed postoperative complications of full or partial flap necrosis. In all patients the perforator marked with Doppler ultrasound was present in each examination in the preoperatively marked spot.

In the case of static thermography the image did not render an unequivocal indication of where a perforator is located in the periods from b1 to b4. The thermal image of the flap had poor/homogenous contrast which would not enable indicating hot-spots. The hot-spots were visible in the location of marked perforators as late as 7 days after the procedure. We associate the result with increasingly stable circulation on subsequent postoperative days, increased vascular flow through perforators and the fact that the flap did not develop collateral circulation on the level of intradermal vascular plexus.

\begin{tabular}{|c|c|c|c|c|c|c|c|c|}
\hline \multirow{2}{*}{$\begin{array}{l}\text { Patient } \\
\text { number }\end{array}$} & \multirow{2}{*}{$\begin{array}{c}\text { Examination } \\
\text { number }\end{array}$} & \multirow{2}{*}{$\begin{array}{l}\text { Number of } \\
\text { Doppler } \\
\text { ultrasound } \\
\text { perforators }\end{array}$} & \multirow{2}{*}{$\begin{array}{l}\text { Doppler } \\
\text { ultrasound } \\
\text { perforators } \\
\text { confirmed by } \\
\text { thermography }\end{array}$} & \multirow{2}{*}{$\begin{array}{l}\text { Number of } \\
\text { hotspots in } \\
\text { static } \\
\text { thermography }\end{array}$} & \multicolumn{2}{|c|}{ ADT - heating } & \multicolumn{2}{|c|}{ ADT - cooling } \\
\hline & & & & & $\begin{array}{c}\text { dTnorm } \\
\text { hot } \\
\text { spots }\end{array}$ & $\begin{array}{l}\text { t90_10 } \\
\text { cold } \\
\text { spots }\end{array}$ & dTnorm & t90_10 \\
\hline \multirow[t]{5}{*}{ IPSI 5} & B1 & 3 & 3 & 11 & 12 & 12 & 12 & 12 \\
\hline & B2 & 3 & 3 & 0 & 5 & 3 & 5 & 3 \\
\hline & B3 & 3 & 3 & 4 & 5 & 6 & 5 & 6 \\
\hline & B4 & 3 & 3 & 4 & 5 & 4 & 6 & 4 \\
\hline & B5 & 3 & 3 & 7 & 5 & 3 & 6 & 4 \\
\hline
\end{tabular}

Tab. 1. Example of Doppler ultrasound perforator examination compared with static and ADT results. 


\subsection{1/qirt.2016.059}

In all dTnorm dynamic thermograms after the flap was stimulated with high temperature hot-spots were consistent with Doppler ultrasound results. The situation was similar in the case of cold-stress dTnorm. What was visible was the difference in the number of hot-spots, which was smaller in the case of heat stimulation than in the cold-stimulated flaps. We associate it with the fact that after the flap is evenly heated and entered into an isothermal state a thermographic camera can only capture the strongest perforators after 180 seconds of observation. We do not rule out that we could have located more hot-spots if the observation period had been longer. We cooled the area down by 5-6 Celsius degrees and we could heat up by max. 5-6 Celsius degrees not to burn the patient although the amplitudes were the same, a smaller heating stimulation amplitude produced a poorer signal in relation to measurement noise.

In testing dynamic thermography t90_10 the perforators marked in Doppler ultrasound were visible after the flap was stimulated with high and low temperature, but due to the noise in the image, the interpretation was difficult and poorly useful in clinical assessment.

The dynamic thermography results described above concerned each test from b1 to b5. Additional hotspots which were visible in dynamic thermography but had not been preoperatively marked in Doppler ultrasound were not verified with Doppler ultrasound at any stage of the investigation. As instant calculation of parametric images was not possible, the sequences were calculated later.

\section{Conclusions:}

Based on our results we think that static thermography is the least useful test for perforator location and we think that cold and heat stress dynamic thermography is a useful test for perforator location, which reduces the time of perforator detection and can be performed at each stage of surgical management, including an intraoperative, non-invasive and repeatable test. In Active Dynamic Thermography dTnorm examination is more precise and easier to interpret than the t90_10 examination. A further investigation is recommended into dynamic thermography to enable differentiation between clinically significant and insignificant perforators.

\section{REFERENCES}

[1] Moon HK, Taylor GI. "The Vascular Anatomy of Rectus Abdominis Musculocutaneous Flaps Based on the Deep Superior Epigastric System”. Plast Reconstr Surg Vol. 82, No. 51998.

[2] Creech, B., and Miller, S. "Evaluation of Circulation in Skin Flaps". In W. C. Grabb and M. B. Myers (Eds.), Skin Flaps. Boston: Little, Brown, 1975

[3] Hartrampf CRJr, Scheflan M, Black PW. "Breast reconstruction with a transverse abdominal island flap". Plast Reconstr Surg. 69;216-225, 1982.

[4] Goodwill RC, Brooks PG. "Abdominal wall hot spots in pregnant women”. J Reprod Med ; 32; 177-180, 1987.

[5] Francis JE, Roggli RI, Love TJ. "Thermography as means of blood perfusion measurement". J Biomech En g; $101 ; 246-251,1979$

[6] Louis de Weerd, MD, Sven Weum, MD, James B. Mercer. "The Value of Dynamic Infrared Thermography (DIRT) in Perforator Selection and Planning of Free DIEP Flaps". Annals of Plastic Surgery, Volume 63, Number 3, September 2009

[7] Yezen Sheena, Toby Jennison, Joseph T. Hardwicke, O. Garth Titley. "Detection of Perforators Using Thermal Imaging". Plastic and Reconstructive Surgery Volume 132, Number 6 December 2013

[8] Nowakowski A., Siondalski P., Moderhak M., Kaczmarek M. A new diagnostic method for evaluation of cardiosurgery wound healing. Quantitative InfraRed Thermography Journal 09/2015. 